\title{
Kinetic Coefficient for Hard-Sphere Crystal Growth from the Melt
}

\author{
Majeed Amini \\ Department of Physics, University of Kansas, Lawrence, Kansas 66045, USA \\ Brian B. Laird \\ Department of Chemistry, University of Kansas, Lawrence, Kansas 66045, USA
}

(Received 12 June 2006; published 20 November 2006)

\begin{abstract}
Using molecular-dynamics simulation, we determine the magnitude and anisotropy of the kinetic coefficient $(\mu)$ for the crystal growth from the melt for the hard-sphere system through an analysis of equilibrium capillary fluctuations in interfacial height. We find $\mu_{100}=1.44(7), \mu_{110}=1.10(5)$, and $\mu_{111}=0.64(3)$ in units of $\sqrt{k_{B} /\left(m T_{m}\right)}$, where $k_{B}$ is Boltzmann's constant, $m$ is the particle mass, and $T_{m}$ is the melting temperature. These values are shown to be consistent, with some exceptions, with those obtained in recent simulation results a variety of fcc metals, when expressed in hard-sphere units. This suggests that the kinetic coefficient for fcc metals can be roughly estimated from $C \sqrt{R /\left(M T_{m}\right)}$, where $R$ is the gas constant, $M$ is the molar mass, and $C$ is a constant that varies with interfacial orientation.
\end{abstract}

PACS numbers: 68.08.De, 02.70.Ns, 81.10.Aj

The kinetic coefficient, $\mu$, of a crystal-melt interface is the constant of proportionality between the growth velocity $(v)$ and undercooling $\left(\Delta T=T_{m}-T\right)$

$$
v=\mu \Delta T
$$

where $T_{m}$ is the melting temperature. Both the magnitude and orientation dependence (anisotropy) of $\mu$ are crucial factors in determining the crystallization rates and growth morphologies of metals [1]-especially for dendritic growth [2]. Because the crystal-melt interface lies between two condensed phases, experimental measurements of $\mu$ are difficult [3] (especially for the anisotropy) and exist only for a few materials, such as $\mathrm{P}_{4}$ [4], and $\mathrm{Pb}$ [5]. The lack of experimental data enhances the role of atomistic simulation, which has been used recently to determine $\mu$ for a variety of systems ranging from Lennard-Jones (LJ) $[3,6,7]$ to close-packed metals [8-10].

Unlike molecular and covalent network materials, the growth kinetics of simple monatomic, close-packed crystals (e.g., simple metals) are not believed to be thermally activated [11]. This view is supported by the results of Broughton, Gilmer, and Jackson (BGJ) [6], who, in a molecular-dynamics (MD) simulation of the fcc (100) interface of a LJ system, found significant crystallization rates even at very low temperatures where the liquid diffusivity is negligible, contrary to the predictions of the thermal activation model of Wilson and Frenkel (WF) $[12,13]$. Based on their MD results, BGJ modified the diffusion-limited WF model to create a collision-limited model in which temperature, not the diffusion constant, plays a central role in the crystal growth of metals. The BGJ model has been interpreted to predict a proportionality between $\mu$ and the interplanar spacing, $d_{l m n}$ for a given interfacial orientation $(l, m, n)$ [14]. Thus, based on relative interplanar spacings, the BGJ model would predict that $\mu_{111}>\mu_{100}>\mu_{110}$ for fcc-forming materials. This prediction works well for (100) and (110) interfaces, where the ratio $\mu_{100} / \mu_{110}$ is often quite close to the BGJ prediction of $\sqrt{2}$, but fails for (111), which is seen in simulations [14] to have the smallest $\mu$ of the three interfaces, not the largest, as predicted by BGJ. The low value of $\mu$ for the (111) interface in fcc systems has been attributed to the formation of transient hcp stacking faults [7,14].

In this work, we determine, via MD simulation, the kinetic coefficient for the hard-sphere system - a standard reference model for close-packed materials. The potential energy of any realizable hard-sphere configuration is zero; therefore, the thermodynamics and kinetics of phase transitions in the hard-sphere system are entirely entropic. Thus, the hard-sphere model can be used to understand the role of entropic driving forces in solidification. It has been shown that the hard-sphere model gives a quantitative description of the crystal-melt interfacial free energy $\gamma$ for simple close-packed systems [15]. Here, we examine the degree to which this is true for the kinetic coefficient.

Recently, two MD simulation methods have been developed to determine $\mu$ and its anisotropy for pure materials: the free solidification method (FSM) and the capillary fluctuation method (CFM). In the FSM $[6,9,16]$, the solid-liquid interface is first equilibrated at the melting point. Next, the system is simulated at a variety of different undercoolings and the interfacial position is monitored. From this data the interfacial velocity, $v$, is determined as a function of the undercooling $(\Delta T)$, from which $\mu$ is calculated using Eq. (1). In the CFM [17], $\mu$ is determined from equilibrium fluctuations of interfacial position. The CFM was chosen for this study because the event-driven nature of hard-sphere MD simulations makes difficult the use of isothermal-isobaric simulation techniques, required for the FSM. A detailed review of these two methods can be found in Ref. [17].

To implement the CFM, separate equilibrium samples of crystal and melt are constructed at the equilibrium coexistence densities. These two samples are then conjoined in 
a single simulation box, which is then equilibrated to form a stable crystal-melt interface. (The procedures for the construction of well-equilibrated crystal-melt interfaces have been outlined in Ref. [18].) For the CFM, a slab geometry is used for the simulation box [19], in which the longest direction is perpendicular to the average interfacial plane. Of the two directions parallel to the interfacial plane, one (defining the width $W$ ) is about one half of the length of the longest direction, and the other (defining the thickness $b$ ) is only a few atomic layers in length, making the interfacial position, $h(x, t)$, a quasi-one-dimensional function of the distance, $x$, along the width of the box. We define $\tilde{h}(q, t)$ as the spatial Fourier transform of the interface height $\left[h(x, t)=\sum_{q} \tilde{h}(q, t) \exp (i q x)\right]$. In the CFM [14], the kinetic coefficient for a particular interfacial orientation is calculated by first measuring the decay time constant, $\tau$, of the time autocorrelation functions of $\tilde{h}(q, t)$ :

$$
\left\langle\tilde{h}(q, t) \tilde{h}^{*}(q, 0)\right\rangle=\left\langle|\tilde{h}(q)|^{2}\right\rangle \exp \left(-\frac{t}{\tau}\right) .
$$

The kinetic coefficient, $\mu$, is related to the decay time constant by

$$
\tau=\frac{1}{\Gamma \mu q^{2}} .
$$

The capillary length, $\Gamma$, is given by

$$
\Gamma=\tilde{\gamma} T_{m} / L,
$$

where $\tilde{\gamma}$ is the interfacial stiffness and $L$ is the latent heat of fusion per unit volume. By determining $\tau$ for several $q$ values for a given interfacial orientation, the product, $\Gamma \mu$, for the interface can be obtained from a plot of $\tau^{-1}$ versus $q^{2}$. The final determination of $\mu$ requires a separate calculation of the interfacial stiffness, $\tilde{\gamma}$. Within the CFM, $\tilde{\gamma}$ can be found from the static autocorrelation function of interfacial fluctuations [20] using the relation,

$$
\left\langle|\tilde{h}(q, 0)|^{2}\right\rangle=\frac{k_{B} T_{m}}{b W \tilde{\gamma} q^{2}},
$$

where $k_{B}$ is Boltzmann's constant. The value of $\tilde{\gamma}$ can then be determined from a log-log plot of $\left\langle|\tilde{h}(q)|^{2}\right\rangle$ versus $q$. The interfacial stiffness is related to the standard interfacial free energy, $\gamma$, by $\tilde{\gamma}=\gamma+d^{2} \gamma / d \theta^{2}$, where $\theta$ is the angle between the interface normal and the (100) direction.

To implement the CFM for hard spheres, we first create equilibrated crystal-melt interfacial samples for three different orientations: (100)[010], (110)[110], and (111) $\times$ [1110]. [ $(h k l)$ refers to the interface normal $(z)$ and $[l m n]$ refers to the short direction $(y)$ of the simulation box.] The values of the coexisting densities for the crystal $\left(\rho_{c}\right)$ and fluid $\left(\rho_{f}\right)$ are $1.037 \sigma^{-3}$ and $0.938 \sigma^{-3}$, respectively [18]. The pressure at coexistence is $11.57(3) k_{B} T \sigma^{-3}$. The thickness, $b$, of the system is as small as 3-5 unit cells to minimize the number of atoms, increase the interfacial fluctuation, and make the interfacial position quasi-one- dimensional. The interfacial width, $W$, is at least $10-15$ times longer than $b$ to give adequate wave-number range for the calculations. Periodic boundary conditions were used, which generates two crystal-melt interfaces in the simulation box. The length of the interface normal is almost twice as long as $W$ to ensure independence of the two crystal-melt interfaces.

After equilibration for $4.2 \times 10^{4}$ time units [for hard spheres, the time unit is $\sigma\left(m / k_{B} T\right)^{1 / 2}$, where $m$ is the particle mass], samples for each interfacial orientation were simulated for $9.8 \times 10^{3}$ time units. (To perform the simulations we use the algorithm of Rappaport [21].) Configurations were recorded every 3.3 time units, generating 3000 configurations for analysis. To determine the interfacial position, $h(x, t)$, for each configuration, it is necessary to define an order parameter $\phi$ to distinguish between solidlike and liquidlike particles. In this work, we use a local order parameter proposed by Morris [22], as modified in Ref. [19]. For each configuration, two interfacial position functions, $h(x, t)$, are determined (one for each interface). For each of the $6000 h(x, t)$ data sets, the

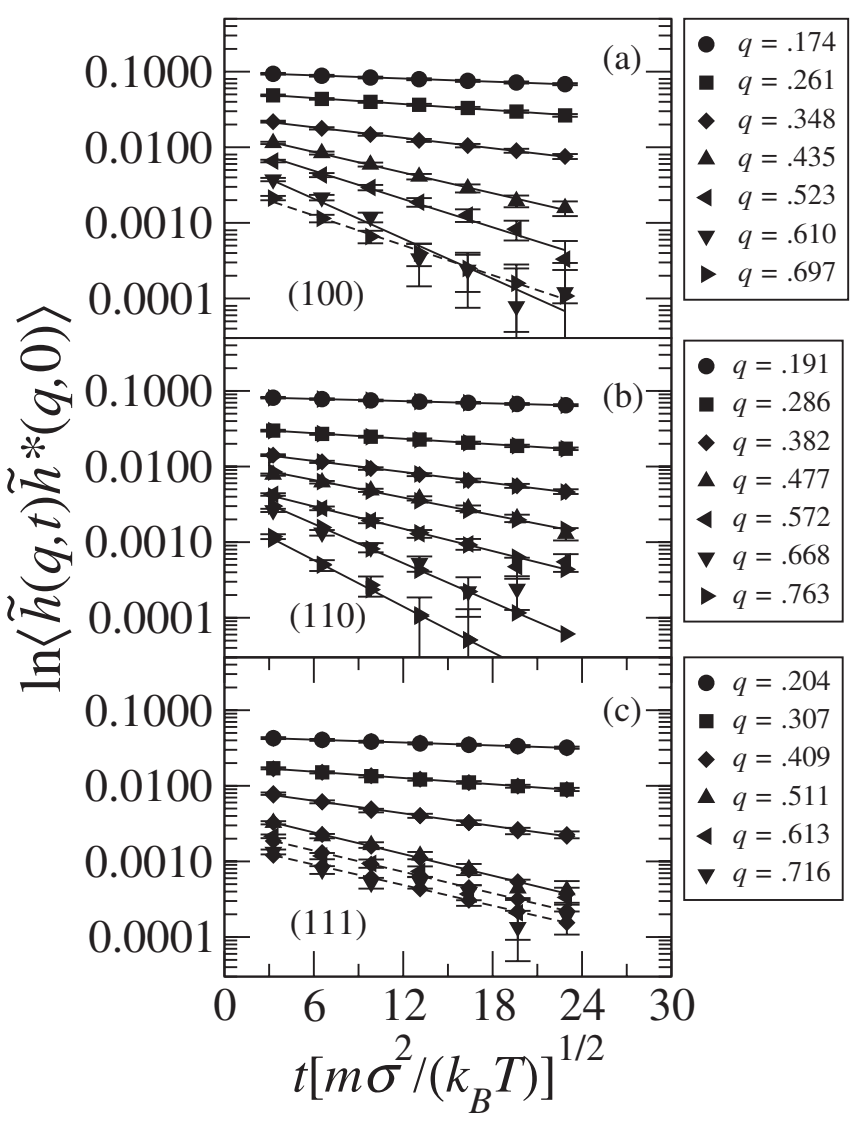

FIG. 1. Plot of $\ln \left\langle\tilde{h}(q, t) \tilde{h}^{*}(q, 0)\right\rangle$ vs $t\left[m \sigma^{2} /\left(k_{B} T\right)\right]^{1 / 2}$ at a variety of $q$ values for (a) (100), (b) (110), and (c) (111). The lines are obtained from weighted linear regression fit. (The dotted lines represent $q$ values not used in the final determination of $\mu$-see text). 
Fourier amplitude, $\tilde{h}(q, t)$, was determined using Fourier transform.

Using Eq. (5) we can determine the interfacial stiffness, $\tilde{\gamma}$, for each orientation from the $y$ intercept of a plot of $\ln \left\langle|\tilde{h}(q, 0)|^{2}\right\rangle$ versus $\ln q$. From this procedure, we obtain stiffness values of 0.425(10), 0.410(16), and 0.74(3) (in units of $k_{B} T / \sigma^{2}$ ) for the (100)[010], (110)[010], and (111)[1 $1 \overline{1} 0]$ interfacial orientations. In addition to the calculation of $\mu$, these values of $\tilde{\gamma}$ can be used to estimate the interfacial free energy, $\gamma$. Using the methods of Ref. [23], we calculate the $\gamma$ to be $0.593(17), 0.572(16)$, and 0.559 (16) for the (100), (110), and (111) interfacial orientations. These values agree (within the error bars) with those previously reported using both the cleaving method [24] and the CFM [19], giving us confidence that we are applying the CFM correctly to this system. We determine $\left\langle\tilde{h}(q, t) \tilde{h}^{*}(q, 0)\right\rangle$ using our data set by averaging over all possible time origins. Following Eq. (2), the values of $\tau^{-1}$ for a variety of $q$ values for each interface are obtained by error-weighted least-squares linear regression from plots of $\ln \left\langle\tilde{h}(q, t) \tilde{h}^{*}(q, 0)\right\rangle$ versus $t\left[m \sigma^{2} /\left(k_{B} T\right)\right]^{1 / 2}$ (Fig. 1). From Eq. (3), we can obtain the product of $\mu$ and the capillary length, $\mu \Gamma$, from the slope of a plot of $\tau^{-1}$ versus $q^{2}-$ shown for all three interfacial orientations in Fig. 2. The circled points at large $q$ where the CFM exhibits significant deviations from linearity (especially for the (111) orientation where stacking faults strongly affect the fluctuations at small length scales) were not included in the determination of $\mu \Gamma$.

To obtain $\mu$ from $\mu \Gamma$, we need to determine $\Gamma$, which is given in Eq. (4) in terms of $\tilde{\gamma}$ and $L / T_{m}$. For hard spheres $L=P\left(\rho_{c} / \rho_{f}-1\right)$, so using the coexistence conditions, we have $L / T_{m}=1.22 k_{B} \sigma^{-3}$. For hard spheres, the natural units for $\mu$ are $\left[k_{B} /\left(m T_{m}\right)\right]^{1 / 2}$. From our data, we obtain values of $\mu$ in hard-sphere units of 1.44(7), 1.10(5), and $0.64(4)$ for the (100), (110), and (111) interfaces, respectively. These values differ in magnitude and relative order

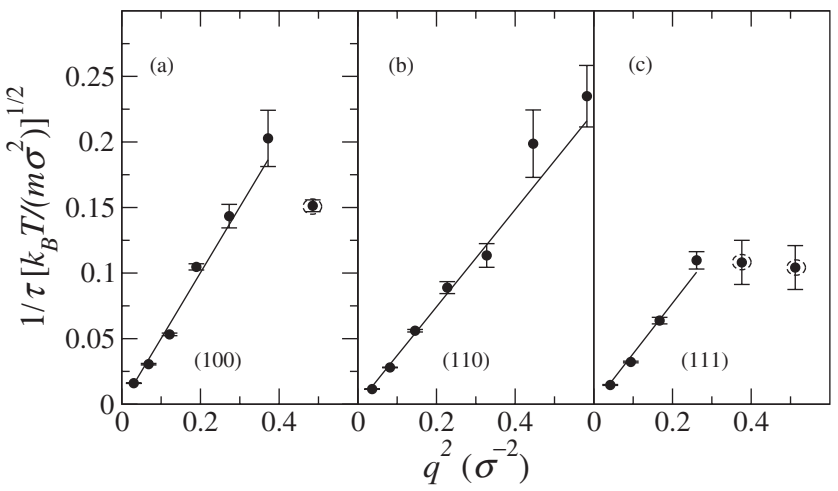

FIG. 2. Plot of $\tau$ versus $q^{-2}$ for (a) (100), (b) (110), and (c) (111) orientations. The lines are obtained from a weighted linear regression to determine the value of $\mu$ using Eq. (4) and (5). The circled points represent data that was excluded from the calculation of $\mu$. from those estimated by Mikheev and Chernov [25] using density functional theory (DFT), which are $0.92,0.65$, and 0.71 in hard-sphere units for the (100), (110), and (111) interfaces, respectively.

Table I summarizes our results for $\mu$ for hard spheres, as well as values from simulation and experiment for a variety of metals. To facilitate comparison, the values for the other systems were converted to hard-sphere units using appropriate values of $T_{m}$ and $m$.

For the hard-sphere system, we find $\mu_{100}>\mu_{110}>$ $\mu_{111}$, in agreement with simulations for the metals $\mathrm{Cu}$ [8], Ni [33,35], Au [10,33,36], and Al [33]. This ordering differs from the DFT prediction of Mikheev and Chernov [25] in which (110), not (111), is the orientation with the lowest value of $\mu$. To quantify the anisotropy, we determine the ratio $\mu_{100} / \mu_{110}$, which for the hard-sphere system is 1.31(9). This value is consistent with the simulations on $\mathrm{Ni}, \mathrm{Au}, \mathrm{Al}$, and $\mathrm{Fe}$, shown in Table I, as well as with the predictions of DFT $[14,25]$ and the interpretation of the BGJ model [14] in which $\mu$ is proportional to the interplanar spacing giving $\mu_{100} / \mu_{110}=d_{100} / d_{110}=\sqrt{2}=$ 1.414. We determine the ratio $\mu_{100} / \mu_{111}$ for the hardsphere system to be 2.26(17), which is at least 50\% larger than the values for the metal simulations listed in Table I, with the exception of $\mathrm{Cu}$ and $\mathrm{Au}$. All results from simulation deviate significantly from the prediction based on lattice spacing $\left(\mu_{100} / \mu_{111}=0.866\right)$, but those for the metals are in good agreement with the DFT [25] prediction (1.29). The deviations of $\mu_{111}$ values from the BGJ predictions have been attributed to "stacking fault drag" $[7,14]$, where crystallization is hindered by the formation of hep stacking faults that must anneal out during crystallization, slowing down (111) growth. This gives a possible

TABLE I. Comparison of our results of $\mu$ for hard spheres with a variety of previous results. All values are reported in hardsphere unit $\left(k_{B} / m T_{m}\right)^{1 / 2}$. The following abbreviations refer to potential models used in the simulations: Foiles, Baskes, and Daw (FBD) [26], Lim, Ong, and Ergolessi (LOE) [27], Voter and Chen (VC) [28], Ergolessi, Parrinello, and Tossati (EPT) [29], Sturgeon and Laird (SL) [30], Ackland, Bacon, Calder, and Harry $(\mathrm{ABCH})$ [31]. The values for $\mathrm{Ni}(\mathrm{FBD})$ and $\mathrm{Au}(\mathrm{VC})$ differ slightly from the published values $([14,32])$ and represent the latest refined values [33]).

\begin{tabular}{llllll}
\hline \multicolumn{1}{c}{ System } & $\mu_{100}$ & $\mu_{110}$ & $\mu_{111}$ & $\frac{\mu_{100}}{\mu_{110}}$ & $\frac{\mu_{100}}{\mu_{111}}$ \\
\hline $\mathrm{HS}$ & $1.44(7)$ & $1.10(5)$ & $0.64(4)$ & $1.31(9)$ & $2.25(18)$ \\
$\mathrm{Pb}($ exp.) [5] & 1.1 & $\ldots$ & $\ldots$ & $\ldots$ & $\ldots$ \\
$\mathrm{Pb}(\mathrm{LOE})[33]$ & $1.07(14)$ & $\ldots$ & $0.90(14)$ & $\ldots$ & $1.2(3)$ \\
$\mathrm{Ni}(\mathrm{FBD})[33]$ & $1.25(8)$ & $0.89(6)$ & $0.84(14)$ & $1.41(13)$ & $1.5(3)$ \\
$\mathrm{Ni}(\mathrm{VC})[33]$ & $1.28(12)$ & - & $0.86(5)$ & & $1.48(17)$ \\
$\mathrm{Cu}(\mathrm{FBD})[8]$ & 1.47 & 0.86 & 0.61 & 1.71 & 2.41 \\
$\mathrm{Au}(\mathrm{VC})[33]$ & $1.31(7)$ & $0.8(2)$ & - & $1.6(4)$ & - \\
$\mathrm{Au}(\mathrm{EPT})[10]$ & $1.06(6)$ & $0.71(6)$ & $0.39(6)$ & $1.49(15)$ & $2.7(4)$ \\
$\mathrm{Al}(\mathrm{SL})[33]$ & $0.84(8)$ & $0.59(6)$ & $0.53(11)$ & $1.42(17)$ & $1.7(4)$ \\
$\mathrm{Fe}(\mathrm{ABCH})[34]$ & $0.84(6)$ & $0.67(8)$ & $\ldots$ & $1.25(17)$ & $\ldots$ \\
\hline \hline
\end{tabular}


explanation for the large value of $\mu_{100} / \mu_{111}$ for the hardsphere system, as the fcc-hcp free energy difference is extremely small for hard-sphere system $[37,38]$.

The data here suggest that a hard-sphere model can account for $60 \%-90 \%$ of the value of $\mu$ for simple fcc metals, implying that the dominant contribution to $\mu$ is entropic in origin. However, the values of $\mu$ for these fcc metals, expressed in hard-sphere units, exhibit a larger deviation from the hard-sphere model than do the corresponding values for the interfacial free energy, $\gamma$, [15], indicating that details of the potential beyond effective atomic size play a significant role in determining the value of $\mu$ for real systems. Further study of the effect of attraction and soft repulsion on the magnitude and anisotropy of $\mu$ is warranted.

In spite of the deviations from the hard-sphere model for specific systems ( $\mathrm{Al}$ and $\mathrm{Fe}$, for example), the values of the kinetic coefficients, $\mu$, for close-packed metals are, at least qualitatively, similar when expressed in natural hardsphere units. This suggests the following semiempirical "rule of thumb" for estimating $\mu$ for such systems:

$$
\mu=C \sqrt{R / M T_{m}}
$$

where $R$ is the gas constant, $M$ is the molar mass, and $C$ is a constant that depends upon the orientation. Based on the data presented here, rough values for $C_{100}$ and $C_{110}$ are 1.3 and 1.0, respectively. The value for $C_{111}$ exhibits quite a bit of variability, ranging from 0.5 to 0.9 , depending upon the substance. Given the lack of accurate experimental data for $\mu$ for real materials, such a rule of thumb may prove useful in solidification studies.

The authors wish to thank Ruslan Davidchack and Marc Odunlami for kindly providing us with their MD code for hard spheres, and J. J. Hoyt for providing us the latest data for $\mu$ for a variety of fcc metals. In addition, we gratefully acknowledge support from the National Science Foundation under Grant No. CHE0316127. Finally, this work has benefited greatly from the participation of one of the authors (B.B.L.) in meetings sponsored by the Computational Materials Science Network, a Department of Energy funded collaboration.

[1] J. Bragard, A. Karma, Y. H. Lee, and M. Plapp, Interface Sci. 10, 121 (2002).

[2] J. S. Langer, Rev. Mod. Phys. 52, 1 (1980).

[3] W. J. Briels and H. L. Tepper, Phys. Rev. Lett. 79, 5074 (1997).

[4] M.E. Glicksman and R. J. Schaefer, J. Cryst. Growth 1, 297 (1967).

[5] G. H. Rodway and J. D. Hunt, J. Cryst. Growth 112, 554 (1991).

[6] J. Q. Broughton, G. H. Gilmer, and K. A. Jackson, Phys. Rev. Lett. 49, 1496 (1982).
[7] E. Burke, J. Q. Broughton, and G.H. Gilmer, J. Phys. Chem. 89, 1030 (1988).

[8] J. J. Hoyt, B. Sadiqh, M. Asta, and S. M. Foiles, Acta Mater. 47, 3181 (1999).

[9] J. J. Hoyt and M. Asta, Phys. Rev. B 65, 214106 (2002).

[10] F. Celestini and J. M. Debierre, Phys. Rev. E 65, 041605 (2002).

[11] D. Turnbull and B. G. Bagley, in Tretise on Solid-State Chemistry edited by N. B. Hannay (Plenum, New York, 1975), Vol. 5, p. 513.

[12] H. A. Wilson, Philos. Mag. 50, 238 (1900).

[13] J. Frenkel, Phys. Z. Sowjetunion 1, 498 (1932).

[14] J. J. Hoyt, M. Asta, and A. Karma, Mater. Sci. Eng., R 41, 121 (2003).

[15] B. B. Laird, J. Chem. Phys. 115, 2887 (2001).

[16] H. L. Tepper and W. J. Briels, J. Chem. Phys. 116, 5186 (2002).

[17] J. J. Hoyt, M. Asta, and A. Karma, Interface Sci. 10, 181 (2002).

[18] R. L. Davidchack and B. B. Laird, J. Chem. Phys. 108, 9452 (1998).

[19] R. L. Davidchack, J. R. Morris, and B. B. Laird, J. Chem. Phys. 125, 094710 (2006).

[20] J. J. Hoyt, M. Asta, and A. Karma, Phys. Rev. Lett. 86, 5530 (2001).

[21] D. C. Rappaport, The Art of Molecular Dynamics Simulation (Cambridge University Press, New York, 1995).

[22] J. R. Morris, Phys. Rev. B 66, 144104 (2002).

[23] M. Asta, J. J. Hoyt, and A. Karma, Phys. Rev. B 66, 100101(R) (2002).

[24] R. L. Davidchack and B. B. Laird, Phys. Rev. Lett. 94, 086102 (2005).

[25] L. V. Mikheev and A. A. Chernov, J. Cryst. Growth 112, 591 (1991).

[26] S. M. Foiles, M. I. Baskes, and M. S. Daw, Phys. Rev. B 33, 7983 (1986).

[27] C. K. Ong, H. S. Lim, and F. Ergolessi, Surf. Sci. 269270, 1109 (1992).

[28] A. F. Voter and S.P. Chen, in Characterization of Defects in Materials, edited by R. W. Siegel (Materials Research Society, Pittsburgh, 1978), p. 173.

[29] F. Ergolessi, M. Parrinello, and E. Tossati, Philos. Mag. A 58, 213 (1988).

[30] J. B. Sturgeon and B. B. Laird, Phys. Rev. B 62, 14720 (2000).

[31] G. J. Ackland, D. J. Bacon, A. F. Calder, and T. Harry, Philos. Mag. A 75, 713 (1997).

[32] D. Y. Sun, M. Asta, and J. J. Hoyt, Phys. Rev. B 69, 024108 (2004).

[33] J. J. Hoyt (private communication).

[34] D. Y. Sun, M. Asta, and J. J. Hoyt, Phys. Rev. B 69, 174103 (2004).

[35] D. Y. Sun, M. Asta, J. J. Hoyt, M. I. Mendelev, and D. J. Srolovitz, Phys. Rev. B 69, 020102(R) (2004).

[36] M. Asta, D. Y. Sun, and J.J. Hoyt, in Thermodynamics, Microstructure and Plasticity, edited by A. Finel et al. (Kluwer, Boston, 2003).

[37] S. Pronk and D. Frenkel, J. Chem. Phys. 110, 4589 (1999).

[38] A. N. Jackson, A.D. Bruce, and G.J. Ackland, Phys. Rev. E 65, 036710 (2002). 